\title{
HERV-W envelope is significantly expressed in Multiple Sclerosis and causes neuroinflammation in animal models with specific antibody inhibition
} Corinne Bernard*1, Jean-Baptiste Bertrand ${ }^{1}$, Ilias Stefas², Francisco Veas ${ }^{2,3}$, Alois B Lang ${ }^{1}$, I Popa ${ }^{4}$, Kamel Sanhadji ${ }^{5}$, Roberta Mancuso, Marina Saresella, M Clerici, JL Touraine ${ }^{5}$, J Portoukalian ${ }^{4}$ and Hervé Perron ${ }^{1}$

Address: ${ }^{1}$ GeNeuro, Genève, Switzerland, ${ }^{2} \mathrm{APOH}-T e c h n o l o g i e s$, Montpellier, France, ${ }^{3} \mathrm{EA} 4169$ University Claude Bernard, Lyon, France, ${ }^{4}$ Graft Immunology and Immunodeficiencies, Faculty of Medicine Laënnec, Lyon, France and ${ }^{5}$ Molecular Medicine and Biotechnologies, \& Don Gnocchi Foundation, Milan, Italy

* Corresponding author

from Frontiers of Retrovirology: Complex retroviruses, retroelements and their hosts

Montpellier, France. 21-23 September 2009

Published: 24 September 2009

Retrovirology 2009, 6(Suppl 2):P70 doi:10.1 186/1742-4690-6-S2-P70

This abstract is available from: http://www.retrovirology.com/content/6/S2/P70

(c) 2009 Bernard et al; licensee BioMed Central Ltd.

Endogenous retroviruses in the human DNA probably result from an exogenous infection of germ line cells. ERVW family has been determined to have entered the mammalian genome after the speciation of old world monkeys, with initial insertion in Catarrhines about 25 million years ago (Voisset et al., 1999). ERV-W copies are present in old world monkeys, superior primates and in humans (then named HERV-W).

HERV-W encodes and may express an envelope protein (ENV), which activates a pro-inflammatory and autoimmune cascade through interaction with Toll-Like receptor 4 (TLR4) on antigen-presenting cells. The specific association of HERV-W RNA with circulating virion particles (previously named "Multiple Sclerosis associated Retroviral element", MSRV) with Multiple Sclerosis disease, its evolution and prognosis has been repeatedly reported.

The role of certain viral infections as triggers for HERV-W expression has also been characterised. This ENV protein was evidenced by several independent RT-PCR and immunohistological studies in MS brain lesions post-mortem (reviewed in Perron et al. Journal of Neurological Sciences, online May 17, 2009).
Using a specific ELISA for HERV-W ENV protein, we report a significant clustering of ENV antigenaemia in about $75 \%$ of MS sera ex-vivo. Large cohorts of blood donors revealed positive antigenaemia in few healthy individuals only $(\mathrm{p}<0,001)$.

Moreover, HERV-W ENV protein is shown to reproduce the "Experimental Encephalomyelitis" MS animal model (EAE) with important inflammatory demyelination evidenced by MRI and histology, as well as anti-myelin autoimmunity. In this MS model, selected anti-ENV monoclonal antibody (mAb) significantly inhibited clinical symptoms compared to untreated controls. Untreated "ENV EAE" mice all died while treated animal survived.

These results now pave the way to the first clinical assessment of a therapeutic agent targeting a human endogenous retroviral protein in a human disease. This antiENV mAb has now been humanised and is subjected to pre-clinical studies. 